\title{
Middle East and North African Health Informatics Association (MENAHIA):
}

\section{Health Informatics Response to COVID-19 Crisis}

Mahmood Tara ${ }^{1}$, Adel Taweel ${ }^{2}$, Dari Alhuwail ${ }^{3}$, Dena A. Al Thani ${ }^{4}$, Eiman Al-Jafar ${ }^{5}$, Eman Abu Hamra ${ }^{6}$, Hassan Ghazal ${ }^{7}$, Hoda Wahba ${ }^{8}$, Kambiz Bahaadinbeigy ${ }^{9}$, Khalid AlKhalifa ${ }^{10}$, Leena Joseph ${ }^{11}$, Mohamed Zeroug ${ }^{12}$, Mohammad Bani Odeh ${ }^{13}$, Mohammed I. Alhefzi ${ }^{14}$, Mounir Hamdi ${ }^{15}$, Mowafa Househ ${ }^{16}$, Najeeb Al-Shorbaji ${ }^{17}$, Osama El Hassan ${ }^{18}$, Salim

Abdullah Al-Salmi ${ }^{19}$, Tamer Amara ${ }^{20}$, Zoubir Sari ${ }^{21}$

1 Fellow of the International Academy of Health Sciences Informatics, Member of the Iranian Medical Informatics Association, Department of Medical Informatics, Mashhad University of Medical Sciences, Mashhad, Iran

2 Department of Computer Science, Birzeit University, Birzeit, Palestine

3 Department of Information Science, College of Life Sciences, Kuwait University; Kuwait Health Informatics Association, Kuwait

${ }^{4}$ Information and Computing Technology Division, College of Science and Engineering, Hamad Bin Khalifa University, Doha, Qatar

${ }^{5}$ Department of Health Informatics and Information Management, Faculty of Allied Health Sciences, Kuwait University; Kuwait Health Informatics Association, Kuwait

${ }^{6}$ Master of Public Health - Al Quds University, Palestine

7 Moroccan Society for Telemedicine and eHealth, Morocco \& National Center for Scientific and Technical Research, Rabat, Morocco

8 Ain Shams University Virtual Hospital, Cairo, Egypt

9 President of the Iranian Medical Informatics Association, Health Information Technology Department, Kerman University of Medical Sciences, Kerman, Iran

${ }^{10}$ National Health Informatics Committee, Supreme Council of Health, Manama, Bahrain

"Oman College of Health Sciences, Ministry of Health, Muscat, Oman

${ }^{12}$ Société Algérienne de Télémédecine et de eSanté, SATeS, Algeria

${ }^{13}$ Palestine National Institute of Public Health, Ramallah, Palestine

${ }^{14}$ Vice President, Saudi Association for Health Informatics, Saudi Arabia

${ }^{15}$ College of Science and Engineering, Hamad Bin Khalifa University, Doha, Qatar

${ }^{16}$ Fellow of the International Academy of Health Sciences Informatics, Hamad Bin Khalifa University, Doha, Qatar

${ }^{17}$ Fellow of the International Academy of Health Sciences Informatics; President of the eHealth Development Association, Amman, Jordan

${ }^{18}$ Health Informatics \& Smart Health Department, Dubai Health Authority, Dubai, UAE

${ }^{19}$ Oman College of Health Sciences, Ministry of Health, Muscat, Oman

${ }^{20}$ Ain Sham University Virtual Hospital, Cairo, Egypt

${ }^{21}$ Société Algérienne de Télémédecine et de eSanté, SATeS, Algeria

\section{Summary}

The unforeseen pandemic of COVID-19 forced all countries worldwide to appreciate digital solutions and their potential contributions in managing such outbreaks. Countries began to share their knowledge and ongoing experiences on how to employ the latest technologies to trace infected cases, warn people on potential danger, increase social and population awareness, and how to provide effective and efficient telehealth services. MENAHIA (The
Middle East and North Africa Health Informatics Association), established in 2018, as a new IMIA regional chapter which has started regional work in several areas of collaboration and knowledge sharing, particularly focused on the common basis of MENA countries' needs towards health informatics solutions regarding COVID-19 crisis management. This article briefly presents health informatics activities and prospects by countries of the Region with a non-exclusive focus on the COVID-19 pandemic.
Needless to say, not all the countries have reported using this plafform, and some may have reported through other venues.

Keywords

COVID-19, digital health, health informatics, Middle East and North Africa, MENAHIA, International Medical Informatics Association, IMIA, National eHealth societies, Algeria, Bahrain, Egypt, Iran, Jordan, Kuwait, Morocco, Oman, Palestine, Qatar, Saudi Arabia, United Arab Emirates 


\section{Introduction}

The recent novel coronavirus (COVID-19) pandemic urged all nations to employ every possible health information technology to enable digital tracing, social distancing, and telecare services. Although countries across the globe were extremely divided in their digital health preparedness in managing such unforeseen outbreak, almost all eventually benefited from partial to full-country-wide anti-COVID solutions whose evidence of potential contributions were fast spreading across the globe.

So far, those who have succeeded were mostly the ones that have outstanding national health information systems and infrastructure, and especially those who have employed the bright minds and brought innovative and practical solutions to the constantly discovered challenges of the ongoing COVID-19 crisis.

\section{MENAHIA and Regional Digital Health Actions}

The Middle East and North Africa Health Informatics Association (MENAHIA) was established in 2018 as the Chapter of the International Medical Informatics Association (IMIA) to represent the IMIA society members in the Region. MENAHIA exists to further the application of informatics methodology and information technology in the field of health care and biomedical research in the Region. The Region is mainly made of the World Health Organization (WHO) Eastern Mediterranean Region and the countries of North Africa of the WHOAfrican Region. Therefore, MENAHIA is made of the following countries: Afghanistan, Algeria, Bahrain, Djibouti, Egypt, Iran, Iraq, Jordan, Kuwait, Lebanon, Libya, Mauritania, Morocco, Oman, Pakistan, Palestine, Qatar, Saudi Arabia, Somalia, Sudan, Syria, Tunisia, United Arab Emirates, and Yemen. Currently, MENAHIA's member societies include those established in Algeria, Bahrain, Iran, Jordan, Kuwait, Lebanon, Morocco, Oman, Pakistan, Qatar, Saudi Arabia, Syria, Tunisia, and United Arab Emirates. The establishment of MENAHIA came as a result of the recognition that health informatics professionals and associations need to work together to learn from each other's and build on the experience gained in the region. It gives us pleasure to have this contribution to the fourth annual report presented as the regional chapter in the IMIA Yearbook. This year witnessed major developments in addition to the establishment of the MENAHIA discussion group on WhatsApp. In December 2019, the General Assembly of the Association met in Kuwait and elected a new president who will take over during the IMIA General Assembly in November this year in Japan. The election of three prominent members from the Region for the fellowship of the International Academy of Health Sciences Informatics (IAHSI) is a clear indication that membership in the Academy from the Region will contribute to balance the global membership. The contribution of professionals in health informatics (medical informatics, digital health, eHealth, bioinformatics, tele-health) research has become more noticeable especially in international literature.

MENA, like all other parts of the world, has been suffering from the COVID-19 pandemic since December 2019. Total curfew was imposed in many countries of the Region in March 2020. Professionals in the Region have made full use of this opportunity by coming together to share lessons learned and discuss common issues of concern. MENAHIA has collaborated with several national, regional, and international bodies to organize webinars and virtual meetings focusing on human resources in health informatics, telehealth, and telemedicine under COVID-19 conditions, legal and ethical issues in eHealth, and artificial intelligence in health. Over 2000 professionals participated in these events (refer to Annex I). This has provided a golden opportunity for knowledge management, sharing, and cross-fertilization in health informatics applications in the Region.

Following is a snapshot of activities that took place in several MENA countries related to the application of digital health during COVID-19.

\section{Algeria}

Algeria is the largest African country in the region, but $80 \%$ of the territory consists of the Sahara Desert with an arid climate that is quite challenging to living conditions.
The existing health system coverage looks comprehensive on paper, but in reality, it is somewhat dysfunctional as a whole, arising from challenges related to the geographical vastness of the territory it is meant to cover. Several initiatives promoted an interest in the benefits of telemedicine and eHealth in Algeria within the scientific and medical communities and among governing bodies. It finally gave rise to the Algerian Society of Telemedicine and eHealth (Société Algérienne de Télémédecine et de eSanté, SATeS). SATeS, which positions itself as a national scientific and non-profit association with a mission to contribute to the promotion of, and development of competencies in telemedicine and digital health in Algeria within a national legal and regulatory framework. Considering the scientific, industrial, socio-economic, and cultural contexts of Algeria, SATeS' approach focuses on promoting the adoption of telemedicine and eHealth systems as a means to attain the sought-after efficiencies in using the health infrastructure and human resources capacities for which the state has devoted significant budgets to develop. Last but not least, SATeS aims to develop and promote a broad and fruitful relationship with other national societies worldwide to gain experience and performance.

\section{Bahrain}

Kingdom of Bahrain has utilized a central system (I-Seha) to report all COVID-19 results [1]. All testing stations whether at public or private facilities were all required to report using the central system. The centralized system played a major role in providing all residents with testing options while maintaining the ability to manage the pandemic centrally. The solution was later useful in applying and managing travel testing requirements. The solution was also utilized for vaccine trials volunteer COVID status tracking. The government of Bahrain has also developed a mobile app for tracing COVID positive individuals as the main method for limiting the spread of the virus. The application also serves as a means for booking testing appointments and viewing COVID-19 test results. 


\section{Egypt}

Ain Shams University Virtual Hospital $(\mathrm{AVH})$ is a healthcare and technology hub that operates via its "Treat \& Teach" initiative since 2016. It was imperative to adjust the preexisting telemedicine services to accommodate the increasing numbers and the variety of patients during the COVID-19 pandemic. Patients were triaged into three categories: emergency care, outpatient care, and telemedicine care (synchronous or asynchronous). Asynchronous telemedicine was performed through WhatsApp and Facebook (Facebook, Inc.) while synchronous was through video consults. Video consultations started using Zoom (Zoom Video Communications, Inc.) and currently are via the AVH platform (https://kashf.asuvh. $\operatorname{com} /$ ). The platform is HIPAA (Health Insurance Portability and Accountability Act) [2] compliant and is a dedicated medical platform (electronic medical records [EMR], e-prescription, online booking, WhatsApp customer service, investigation upload, etc.). Guidelines for every specialty have been laid by consultants after revising the appropriate international guidelines. The consultants received virtual training, testing sessions, technical support, and medical decision support before and after the launch of the triaging system. A mobile application to triage COVID and Non-COVID patients is to be launched within a few days providing asynchronous triaging for patients. It is integrated with Kashf (Checking) video-consultation platform and utilizes artificial intelligence to improve its accuracy.

Kashf currently (November/2020) has 1239 bookings and has served patients from 16 countries. Around $4500 \mathrm{CO}$ VID-19 patients were also triaged, and home isolation patients were followed up. Patients were followed up through the WhatsApp numbers regarding new symptoms, the progression of any present symptoms, oxygen saturation, new infected household members, and control of comorbidities. Most of the patients were satisfied with the service and most of the physicians were confident with their telemedicine services.
Iran

Iran started the battle with the COVID-19 crisis using a triple strategy comprising of a national COVID registry, a national COVID hotline, and an MoH-supported mobile application. Such a combination allowed the country to build the first model of the COVID-19 epidemic spread supporting the government decisions on lockdowns and social physical limits. The map was also used to constantly inform all citizens regarding the red zones (highly infected zones).

In addition, the $\mathrm{MoH}$ recently started an initiative to expand and advance the telehealth services aiming at supporting COVID-19 care programmes including teleconsultation, teleradiology, and televisits. Eight medical universities and one mega-region (mega-region 9 including three provinces of Northern, Southern Khorasan, and Khorasan Razavi) were engaged in the pilot programmes. At the same time, the $\mathrm{MoH}$ established the national committee for telemedicine services, developed and announced the national operational policy for telecare services covering several categories including tariffs, certification and accreditation, insurance coverage, security and privacy, standards, knowledge and skill requirements. While the current telecare services are focused on COVID-19 care programmes, essential health care services, particularly for chronic disease patients, are the next priority in the $\mathrm{MoH}$ stepwise strategic plan.

Along with the government's endeavors on COVID-19 outbreak management, a group of fifty Iranian specialists in medical informatics and health information management domains, supported by the Iranian Association of Medical Informatics, voluntarily established a virtual think-tank group to formulate the most practical and up-to-date Information Technology (IT) solutions to help the national and provincial health authorities. As result, the group published several anti-COVID IT solution documents and guides in the areas of digital tracing, telehealth services, and $\mathrm{CO}$ VID management dashboards and indicators, and made them freely available to all health authorities across the country. Those solutions have been used as the basis for many national and provincial actions in the management of the COVID-19 crisis.

\section{Jordan}

Jordan has witnessed several developments in response to the COVID-19 pandemic. Such responses have been in three main lines. The first line was AMAN (Safety in Arabic) App which was developed for Jordan's Ministry of Health $(\mathrm{MoH})$ by COVID-19 JOTECH Community, a group of tech-savvy volunteers. AMAN is a privacy-conscious, exposure detection App that helps keep the community and Jordan safe from coronavirus. AMAN is a community-driven App using anonymous Global Positioning System (GPS) data to detect exposure to Covide-19 patients by storing the log of citizen's location for 14 days. If an AMAN's user is diagnosed with coronavirus by the $\mathrm{MoH}$, all people who have been recently in contact with her/him will be notified through the APP advising them to isolate and call a three-digit local number (COVID-19 Hotline) for instructions. In the second line, $\mathrm{MoH}$ launched an Electronic Services Platform "e-Med", which enables patients registered in the electronic health record application (Hakeem) to request their monthly recurring medications electronically aiming to save time and effort. The platform was developed to support the Jordanian medical sector in light of the current pandemic of which the total curfew is one of them. During the curfew, patients were not allowed to leave their place of residence, and healthcare services were mostly COVID-limited. As the third line, King Abdullah University Hospital launched a free smart application that allows their patients to book appointments, make reservations, and access their medical records including visits, prescriptions, and medical laboratory results.

\section{Kuwait}

Shortly after the first case of COVID-19 was announced in Kuwait, a plethora of digital health efforts from the civil society as well as the public and private sectors were launched to combat COVID-19. The Kuwait Health Informatics Association (KHIA) played a key role in fighting the misinformation infodemic by raising the public's awareness about COVID-19 and sources of reliable information. Additionally, KHIA collabo- 
rated with MENA Telehealth Forum and International Society for Telemedicine and eHealth (ISfTeH) in sponsoring a series of Telehealth webinars targeting various healthcare professionals to introduce the utilization of digital health solutions (e.g. telehealth) against COVID-19. Also, KHIA created a database for members who volunteered to support MoH's digital health efforts. On the government side, the $\mathrm{MoH}$ developed an information system and a mobile app for contact tracing purposes. Multiple healthcare institutions in the public and private sectors have transitioned to Telehealth (e.g. patient visits, Teleconsultation, and Telepharmacy) in an attempt to reduce patient visits and limit exposure to COVID-19. Furthermore, the Kuwait Surgical Telehealth Network (KSTN) has actively leveraged health information technology by connecting 40 operating theaters across 10 government healthcare facilities for live video surgeries and virtual patient monitoring.

Leveraging their digital skills, various volunteer scientists and informaticians contributed to the fight against COVID-19. With more than 52,000+ visits, the CoronaMaps Kuwait [3] leverages an open spatial hub to create, organize, visualize, and share geo-enabled information about COVID-19. Using an anonymous short-form, TrackCOVIDKW.com [4] predicts and locates hotspots by evaluating how many people in the area have developed COVID-19 symptoms. A partnership between developers and certified professionals specialized in mental health, the National Counseling Program for COVID-19 (CoronaCareKW) offers online advice and counseling related to mental health during the COVID-19 pandemic [5]. The programme had more than 30 thousand visitors, offered over 300 support sessions through more than 950 volunteers.

\section{Morocco}

In Morocco, the $\mathrm{MoH}$ and the National Physicians Order (CNOM) launched a free telemedicine platform (www.tbib24.com) to allow continuing remote medical care during the lockdown. Similarly, a dozen other "tele-consultation" platforms have been created since the beginning of the pandemic to cut down unnecessary travels. The CNOM has set up a telemedicine commission to update the regulations in telemedicine practices and to establish guidelines. Morocco has established a legal framework for safe telemedicine practices since 2015, however, the pandemics revealed some incoherence that blocked the full deployment of telecare. Furthermore, the $\mathrm{MoH}$ in partnership with the Ministry of Interior, the Digital Development Agency (ADD), and the National Telecommunications Regulatory Agency (ANRT) developed a virus tracking and COVID-19 patients tracing application named "Wiqaytna" (Our Protection in Arabic). To maintain data privacy, the application has undergone assessments by the National Commission for the Control of Personal Data Protection (CNDP) and obtained its prior approval.

\section{Oman}

To tackle the Coronavirus outbreak in the Sultanate, Oman has come up with a technologically significant measure by launching an application named Tarassud (monitor in Arabic) Plus which is integrated with the $\mathrm{MOH}$ national (electronic health record (EHR). It provides up-to-date, transparent COVID-19 statistics, guidelines and best practices. It can be used by the general public for reporting illness and is available in five different languages namely Arabic, English, Hindi, Bangladeshi and Urdu.

The system allows quarantining a patient at home or any designated institutions specified by $\mathrm{MOH}$. It includes the facility to track the symptoms of the quarantined persons and their physical location using a wrist band. Through the management board, the system provides a graphical representation of registered cases, making it easier for the user to track the status of the case, the movement of the patient, and related entities. It also allows the airport staff to register arriving passengers.

Tarassud Plus has a dashboard wherein one can find the total number of cases worldwide depicted in diagrams. Moreover, it has a medical scout page which is a chatbot service wherein a user can post their update status and diagnosis with support of the artificial intelligence mechanism, and the admin user can easily track the status of the patient and render necessary aid. The indicators from the application have helped in making informed decisions, thereby curbing the pandemic (and taking appropriate measures as required / and improving treatment outcome) [6-10].

\section{Palestine}

Several initiatives in the use of health informatics solutions were developed as a response to the COVID-19 crisis. One initiative was the launch of a toll-free hotline to provide medical consultation to COVID-19 cases and to inform the Ministry of Health $(\mathrm{MoH})$ about potential cases. $\mathrm{MoH}$ reported the receipt of 250,000 calls since the outspread of COVID-19 in the local community in Gaza. To record and monitor the spread of COVID-19 in Palestinian towns and cities, the $\mathrm{MoH}$ developed a national surveillance system [11]. The system provides the latest statistics on the number of active infections, death rates and cured cases. It also provides statistical prediction of COVID-19 infections spread and potential hotspots.

Another initiative by the $\mathrm{MoH}$ was the launch of telemedicine services as a response to the closure of the primary health centers after the discovery of the first COVID-19 cases. A similar initiative, UNRWA "Toll-free telemedicine hotlines", was additionally provided to support UNRWA's primary healthcare centers [12]. The $\mathrm{MoH}$ also provided several advanced telemedicine services that included medical teleconsultations, health education, and psychosocial support. Recently, the $\mathrm{MoH}$ launched a mobile application for telemedicine program called "Sehati" (My Health in Arabic) to decrease the load on the hotlines, and to provide teleconsultation services to COVID and non-COVID cases [13]. The app was also used to deliver the results of the Polymerase Chain Reaction (PCR) tests to patients in a more secure and efficient manner. Another complimentary work, by the $\mathrm{MoH}$, included the development of a Family Health e-System to enable providing essential primary health care services, across 360 clinics, including enabling early 
detection of COVID-19 infection. Recently, the government has also initiated the national system for health statistics and data to enable automated collection of data required for the health indicators, from the National Health Information System [13], supported through the Palestinian Health Information Center [14]. These developments are led by the national digital health team, drawn from across the Ministry of Health Information Technology and the Palestinian National Institute of Public Health $[15,16,17]$.

\section{Qatar}

There have been several solutions against COVID-19 in Qatar. In April 2020, the Ministry of Interior launched the EHTERAZ (Caution in Arabic) application [18] to provide daily updates about COVID-19. The EHTERAZ application, as a contact-tracing application was made mandatory for each resident to control their movements, which was key to its successful implementation. In addition, a portal running by the Ministry of Public Health (MOPH) provided the public with useful information such as phase lifting restrictions and guidelines for vulnerable populations [19]. In addition, the Hamad Medical Corporation in collaboration with the Ministry of Transport and Communication had launched their remote healthcare services program in March 2019 providing patients with virtual consultations, medical advice, medication delivery, and the issuing of sick leave certificates [20]. Furthermore, Hamad Bin Khalifa University's (HBKU) Human Health and Well-Being Research Group developed pilot applications and conducted webinars to discuss their COVID-19 research. Some of the suggested applications included the WEQAYA (Prevention in Arabic) app and the ESRA (Emotion Sensing Recognition App). As these developments continue to evolve, there would be discussions at the policy level, to keep, maintain, and innovate on the current initiatives to help improving healthcare delivery in the future beyond the COVID-19 pandemic.

\section{Saudi Arabia}

The Kingdom of Saudi Arabia (KSA) ramped up its digital health efforts to battle the COVID-19 crisis. Various digital tools, platforms, and applications were used to assist the Kingdom's public and private healthcare sectors alike. These digital tools, platforms, and applications were used for COVID-19 screening, disease surveillance, contact-tracing, follow-up, as well as raising awareness. For instance, using MoH's available smartphone applications, COVID-19 symptom checker to enable suspected COVID-19 individuals to directly book appointments at dedicated COVID-19 clinics and drive-throughs, via mass testing locations. Additionally, the Health Electronic Surveillance Network (HESN) and others were leveraged as reliable databases for all COVID-19 laboratory tests in KSA [21], whilst other virtual interventions took part with similar nature. On the 11th of August, Riyadh held the Global Digital Health Summit, where the 'Riyadh Declaration on Digital Health' was formulated and issued. The Declaration articulated seven key priorities and nine recommendations for data and digital health that need to be adopted by the global health community to address COVID-19 pandemic challenges, as well as preventing future pandemics [22], Table 1 highlights its key recommendations. On another instance, Saudi Arabia has chaired the 2020 G20 summit, where global subject matter experts pointed out the promise of digital health in the unprecedented times of COVID-19; which was evident in the G20 Riyadh Summit closing remarks [23]. As a result, the G20 Digital Health Taskforce have published different global digital implementations, recommendations and use cases to combat and, hopefully prevent future pandemics [24].

\section{United Arab Emirates}

In contrast to other Gulf Cooperation Council (GCC) countries, the United Arab Emirates has a unique setup for its healthcare sector. The country's healthcare field is regulated through a hybrid structure of both Federal "Country-level" authority which is the Ministry of Health and Prevention "MOHAP" as well as local authorities at the two major Emirates: Abu Dhabi and Dubai. Therefore, one would witness diversified

Table 1 Recommendations from the Riyadh Global Digital Health Summit [23].

1) Implement data-driven and evidence-based protocols for clear and effective communication with common messaging to build citizens' trust.

2) Work with global stakeholders to confront the propagation of misinformation or disinformation through social media platforms and mass media.

3) Implement a standard global minimum dataset for public health data reporting and a data governance structure tailored to communicable diseases.

4) Ensure countries prioritize digital health, particularly, improving digital health infrastructure and reaching digital maturity.

5) Enable health and care organizations by providing the necessary technology to collect high-quality data in a timely way and promote sharing to create health intelligence.

6) Cultivate a health and care workforce with the knowledge, skills, and training in data and digital technologies required to address current and future public health challenges.

7) Ensure surveillance systems combine an effective public health response with respect for ethical and privacy principles.

8) Develop digital personal tools and services to support comprehensive health programs (in disease prevention, testing, management, and vaccination) globally.

9) Maintain, continue to fund, and innovate surveillance systems as a core component of the connected global health system for rapid preparedness and optimal global responses. 
digital health transformation initiatives across these healthcare jurisdictions during the last 10 years. However, three distinctive themes of healthcare informatics-intensive projects, which are strongly backed by regulatory framework and public-private partnership engagements, have prevailed in the last 18 months. These themes are public health-related information systems, virtual care initiatives, and Health Information Exchange implementations.

Projects targeting the management of the COVID-19 pandemic such as outbreak management, contact-tracing, and quarantine procedures have become national priorities since March 2020. MOAHP has put forward an information/command center, several mobile apps to offer critical functionalities e.g., public awareness, symptoms checkers, quarantine's virtual assistant, contact-tracing, and many other related services. Other local authorities have also spent great efforts to complement the work of MOHAP in their jurisdictions.

The pandemic has radically boosted the adoption of virtual care practices across the healthcare ecosystem. Therefore, healthcare and non-healthcare regulators exhibited agility and responsiveness by relaxing the existing restrictions and governing policies of these practices such as the medical liability law [25] and the standards for telehealth services [26].These regulations and standards aim at regulating the provision of virtual care medical services, which are witnessing a great advancement worldwide. As a result, a number of governmental virtual care projects were disseminated such as MOAHP's virtual clinics and Dubai Health Authority's initiative of "Doctor for Every Citizen", and "Abu Dhabi's Telemedicine Center".

\section{Conclusion}

Although MENAHIA has recently started to evolve, the recent evidence of regional collaboration and event series along with countries' timely health informatics responses has demonstrated big potentials in this IMIA young partner. With further support from IMIA, it is expected that member societies of the MENA region play a more significant and effective role in their continuing co-battle against COVID-19 and after through constant collaboration and knowledge sharing.

\section{Annex I: List of main events held in the Region}

1. Regulatory and data requirements around telehealth during and post pandemic, 22 April 2020

2. Digital Transformation in the Midst of COVID-19: Learning from NYC's Frontliners, 12 May 2020

3. MENA Telehealth Majlis, Bahrain, 15 May 2020

4. MENA Telehealth Majlis, Jordan, 4 June 2020

5. MENA TELEHEALTH Majlis, Kuwait, 2 July 2020

6. MENA Telehealth: Practical and clinical considerations for tele-oncology, 3 June 2020

7. Clinicians Licensing, Continuous Trainings \& Accreditation during and post COVID-19: Telling the digital story, 10 August 2020

8. Effective Pharmacovigilance Outsourcing, 02 September 2020

9. GCC EHEALTH WORKFORCE DEVELOPMENT CONFERENCE, 14 - 16 November 2020

10. The Middle East Telehealth Technology Forum, 24-25 November 2020

11. The 3rd Arab African Telemedicine Conference, 25 November 2020

\section{References}

1. Bahrain Ministry of Health. National Health Information System (I-SEHA) [Internet]; 2020. Available from: https://www.moh.gov.bh/iSeha?lang=en

2. US. Centres for Disease Control and Prevention. Health Insurance Portability and Accountability Act, 1996 (HIPAA); [Internet] 2020. Available from: https://www.cdc.gov/phlp/publications/ topic/hipaa.html

3. CoronaMaps Kuwait [Internet]; 2020. Available from: https://coronamapskw.com

4. COVID-19 Community Tracking Kuwait [Internet]; 2020. Available from: www.trackcovidkw.com

5. CoronaCareKW [Internet]; 2020. Available from: https://coronacarekw.com

6. World Health Organization (WHO). Oman launches technological surveillance system to track COVID-19 [Internet]; 2020. Available from: https://www.who.int/news-room/feature-stories/ detail/oman-launches-technological-surveillan- ce-system-to-track-covid-19

7. World Health Organization (WHO). Using technology to contain COVID-19 in Oman [Internet]; 2020. Available from: http://www.emro.who.int/ health-topics/corona-virus/using-technology-tocontain-covod-19-in-oman.html

8. Times of Oman. Oman's health ministry releases update for Tarassud app [Internet]; 2020. Available from: https://timesofoman.com/article/omans-health-ministry-releases-update-for-tarassud-app

9. Oman Observer. New update of Tarassud + application released [Internet]; 2020. Available from: https://www.omanobserver.om/new-update-of-tarassud-application-released/

10. Sadek G. Saudi Arabia; Oman; Kuwait: Ministries of Health Begin Using Mobile Apps to Combat COVID-19 [Internet]. The Library of Congress; 2020. Available from: https://www.loc.gov/law/ foreign-news/article/saudi-arabia-oman-kuwaitministries-of-health-begin-using-mobile-apps-tocombat-covid-19/

11. Palistinian Ministry of Health. CORONAVIRUS-COVID-19 Surveillance system [Internet]; 2020. Available from: http://site.moh.ps/index/ covid19/LanguageVersion/1/Language/ar

12. The Palestinian Information Center. "103" Free service to combat Corona in Gaza. The Palestinian Information Center. [Internet]. 2020 Sept 3. [cited 2020 Oct 20].Available from: https://tinyurl.com/ y6ryebzr

13. Palistinian Ministry of Health. Sehatty [Mobile app]; 2020. Available from: http://apps.moh.gov. ps/sehatty

14. IntraHealth International. Palestinian Ministry of Health Saves Mmoney, Improves Quality of Care Through Its Health Information System: Palestinian Health Capacity Project (PHCP) [Internet]; 2018. Available from: https://www.intrahealth.org/ sites/ihweb/files/attachment files/phcphistubasimplementation.pdf

15. United Nations Economic and Social Commission for Western Asia. Palestinian Health Information Center [Internet]; $\mathrm{n} / \mathrm{d}$. Available from: https://www. unescwa.org/palestinian-health-information-center

16. World Health Organization (WHO). The Palestinian National Institute of Public Health [Internet]; $n / d$. Available from: http://www.emro.who.int/pse/programmes/opt-national-institute-public-health.html

17. The Palestinian National Institute of Public Health [Internet]; $\mathrm{n} / \mathrm{d}$. Available from: https://www.pniph. org/en

18. Qatar Ministry of Public Health. Covid-19 portal [Internet]; 2020. Available from: https://covid19. moph.gov.qa/EN/Pages/default.aspx

19. Qatar Ministry of Public Health. Qatar launching new remote healthcare services [Internet]; 2020. Available from: https://www.moph.gov.qa/english/ mediacenter/News/Pages/NewsDetails.aspx?ItemId $=115$

20. Hamad Bin Khalifa University. HBKU's College of Science and Engineering Collaborates with MoTC to Deliver Webinar Series: Online events highlight COVID-19 related research and applications [Internet]; 2020. Available from: https://www.hbku. edu.qa/en/news/cse-collaborates-motc 
21. Hassounah M, Raheel H, Alhefzi M. Digital Response During the COVID-19 Pandemic in Saudi Arabia. J Med Internet Res 2020;22(9):e19338-e.

22. Knawy B, Adil M, Crooks G, Rhee K, Bates D, Jokhdar H, et al. The Riyadh Declaration: the role of digital health in fighting pandemics. Lancet (London, England) 2020;396(10262):1537-9.

23. G20 Health Ministers' Declaration [Internet]; 2020. Available from: https://g20.org/en/media/ Documents/G20\%20Health\%20Ministers $\% 20$ Declaration_EN_\%2020201119.pdf
24. Digital Health Taskforce [Internet]; 2020. Available from: https://digitalhealthtaskforce.org/

25. United Arab Emirates Ministry of Health \& Prevention. Ministry of Health and Prevention Underscores that the Approval of the Executive Regulation of Medical Liability Law Would Strengthen Health [Internet]; 2019. Available from: https://www.mohap.gov.ae/en/MediaCenter/News/ Pages/2106.aspx

26. Dubai Health Authority. Standards for Telehealth Services [Internet]; 2019. Available from: https://
www.dha.gov.ae/Documents/HRD/RegulationsandStandards/standards/Standards $\% 20$ for $\% 20$ Telehealth\%20Services\%20Final.pdf

\section{Correspondence to:}

Dr. Dari Ahuwail

Department of Information Science

College of life Sciences

Kuwait University, Kuwait

E-mail: dari.alhuwail@ku.edu.kw 